\title{
Measurement of hand-vibration transmissibility by non-contact measurement techniques
}

\author{
P. Nataletti ${ }^{1}$, N. Paone ${ }^{2}$ \& L. Scalise ${ }^{2}$ \\ ${ }^{1}$ Centro Ricerche ISPESL, Monteporzio Catone, Roma, Italy \\ ${ }^{2}$ Department of Mechanics, Università Politecnica delle Marche, Ancona, \\ Italy
}

\begin{abstract}
Background: Workers who use hand-held vibrating tools may experience finger blanching attacks due to episodic vasospasm in the digital vessels. In occupational medicine, the pathological consequences to the exposure to handtransmitted vibration are known as vibration induced white finger (VWF). In many cases, workers are recommended to use anti-vibration gloves and a standard procedure (ISO 10819) is used to test and qualify such anti-vibration gloves. Some problems and limits are known for what concerns the measurement and procedure proposed by this standard. Materials and methods: Hand-arm vibration transmissibility (ratio between rms acceleration on different points of the upper side of the hand and the handle rms acceleration) of 13 healthy subjects (main BMI: $24.3\left[\mathrm{~kg} / \mathrm{m}^{2}\right]$ ) was measured using a mono-axial accelerometer on the handle and a laser Doppler vibrometer on several points on the hand. The laser vibrometer eliminates any mass loading effect on the hand tissue. Seven single frequency excitations ( $\mathrm{rms}$ acceleration amplitude: $3 \mathrm{~m} / \mathrm{s}^{2}$ ) have been tested: 15, 20, 30, 50, 70, 90 and $110 \mathrm{~Hz}$. Closed loop control of the handle acceleration is provided. Monitoring of the push force $(50 \pm 5.0 \mathrm{~N})$ has been carried out during the test. Results: Transmissibility higher then one (1.54) has been measured at the tip of the fingers, especially for vibration frequencies lower then $70 \mathrm{~Hz}$, while transmissibility lower then $1(0.20)$ is reported on the hand knuckles for frequencies higher then $30 \mathrm{~Hz}$. Conclusions: The measurement procedure presented a non-contact measurement of the vibration transmissibility of the human hand and in particular its spatial distribution over the hand.

Keywords: hand vibration, transmissibility, laser Doppler vibrometry.
\end{abstract}




\section{Introduction}

Workers operating in industrial environment and assembly lines, which operate vibrating tools, are subjected to long-term vibration exposure. This is demonstrated to be associated to vascular, neurological and muscular-skeletal symptoms and disorders (Griffin, [1]). It has been calculated (Wasserman and Wasserman, [2]) that about 10 million of workers are daily exposed to vibration during their working activities in the USA. It is estimated (Taylor [3]) that a percentage between the $30 \%$ and the $90 \%$ of the exposed population will eventually develop the disorder generally defined as hand-arm vibration syndrome (HAVS). HAVS has been especially associated to the specific vascular symptom called vibration-induced white finger (VWF). Significant positive correlation between VWF and hand-transmitted vibration levels has been demonstrated (Inaba et al. [4]) with prevalence of symptoms among woman respect to male workers (Mirbod et al. [5])).

The use of special anti-vibration gloves is considered a way to protect the worker to the exposure to the vibration. The International ISO 10819 standard was established to set a repeatable procedure to quantify the attenuation efficiency (reduction of the acceleration transmitted to the hand palm) of such gloves. According to some authors the use of such gloves is useful in the reduction of the vibration measured on the hand for specific tools such as the pneumatic chipping hammer (Goel and Rim, [6]). Nevertheless the standard itself underlines the impossibility to determine the transmissibility to the fingers. Some criticisms and "weak points" have been reported (Hewitt, [7]) especially for what concerns:

1 - Test set-up (for example: possible misalignment of the accelerometer adaptor).

2 - The impossibility to determine the transmissibility of the fingers, where the accelerometer cannot be fixed.

3 - The difficulty to carry out the tests, due to the presence of many influencing parameters.

The aim of the present paper is to propose a novel approach for the experimental setup for the assessment of the vibration transmissibility directed to the hand. Indeed such an approach could also be used to assess glove performance (contributing to improve ISO-10819). The proposed approach can provide information on the spatial distribution of the acceleration (and consequently of the vibration transmissibility) over the operators hand during the vibration exposure, without mass loading effect, that would be caused by the use of accelerometers.

\section{Materials and methods}

The testing procedure used in the following is based on ISO 10819. The measurement apparatus is in large part the one proposed in the same standard a part from the vibration transducer used in contact with the hand palm. 


\subsection{Experimental apparatus}

The measurement apparatus realised for the experimental tests is schematically reported in Figure 1. The test bench is composed by: an instrumented platform with a load cell for the measurement of the push force; a electro-dynamic shaker and a closed loop controller in amplitude; a laser Doppler vibrometer; a single axis accelerometer and the signal conditioner and a control/processing unit composed by an A/D 16 bit card and a computer. The optical axis of the laser vibrometer was always aligned with the excitation direction provided by the shaker axis; a mirror was used to guarantee such condition. A detail of the experimental set-up and of the instrumented handle is reported in figure 2. The laser beam can be aimed at different points on fingers keeping the same direction of measurement.

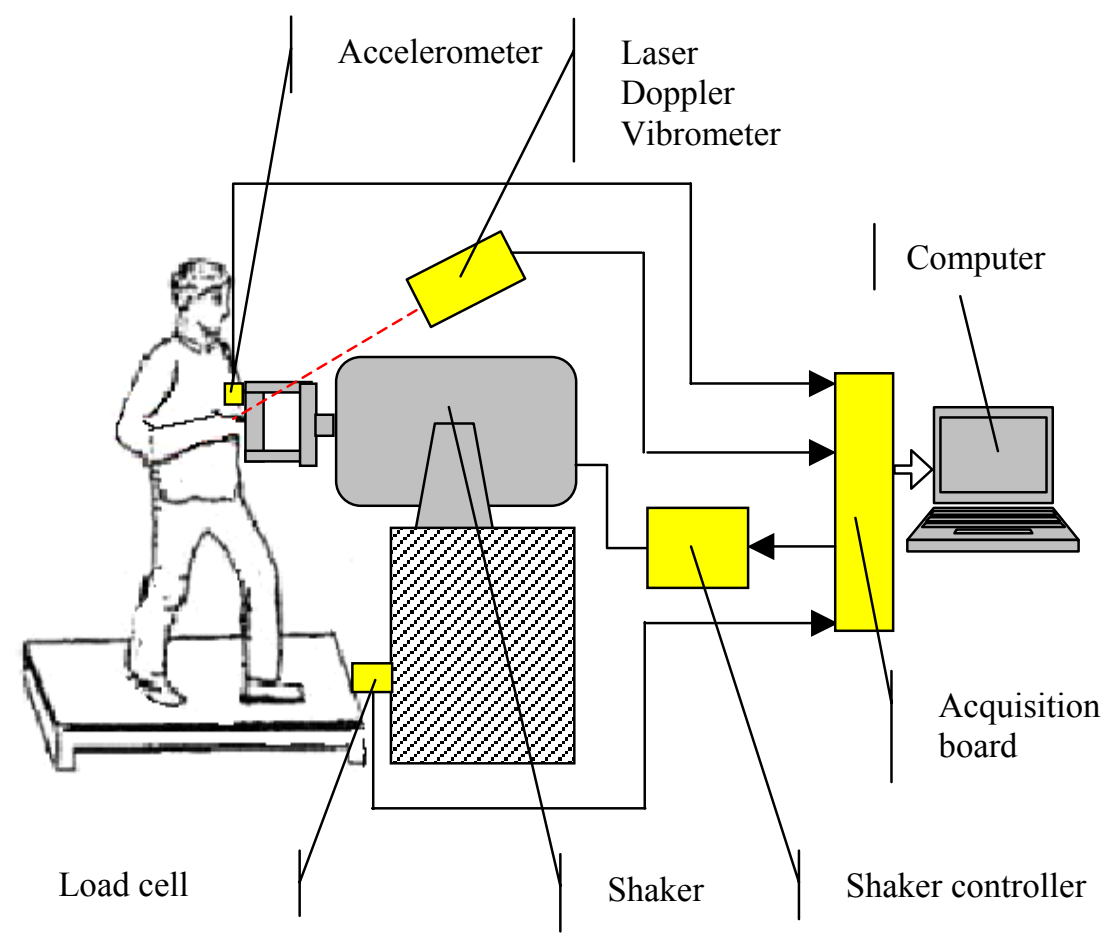

Figure 1: Schematic of the test set-up.

\subsection{Test procedure}

The subjects were instructed to assume correct posture and to exercise the requested push force $(50 \pm 5 \mathrm{~N})$; pieces of retro-reflective tape $(5 \times 5 \mathrm{~mm})$ were attached to the subject hand in order to improve the measurement conditions for the laser vibrometer. Each test had duration of $30 \mathrm{~s}$ and tests were conducted during 4 days and none of the subjects performed more then 4 consecutive tests. 
Each test (test duration: $30 \mathrm{~s}$ ) has been repeated 10 times and average values have been calculated. Seven excitation frequencies were studied: 15, 20, 30, 50, 70, 90 and $110 \mathrm{~Hz}$; acceleration was maintained constant at the handle (acceleration $\mathrm{rms}=3 \mathrm{~m} / \mathrm{s}^{2}$ ) during each test by means of a closed loop controller. The total number of observations for each frequency and for each subject is 120 (12 measurement points, 10 measurements).
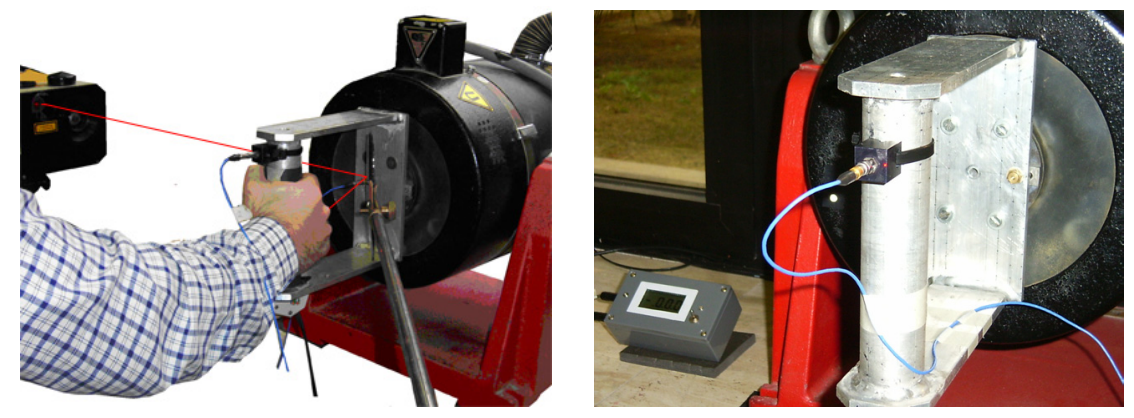

Figure 2: Detail of the test conditions (left); The instrumented handle, the accelerometer and the display for the push force visual control (right).

\subsection{Subjects and investigated variables}

The study was carried out on 13 healthy, male subjects (average BMI =24.3; standard deviation $=0.9$ ) aged 24-31 with no previous significant exposure to vibration. The study participants were recruited among the student population of the university and the technicians of the laboratory.

The transmissibility, $T R$, is calculated from the rms acceleration values as follows:

$$
T R=\text { acceleration measured at point } P_{i, j} / \text { acceleration at the handle; }
$$

Where $P_{i, j}(i=1 \ldots 3$, number of points along each finger; $j=1 \ldots 4$, number of measured fingers) are the 12 measurement points on the hand reported in figure 3.

Each subject, during the tests, maintained the push force at $50 \mathrm{~N} \pm 5 \mathrm{~N}$ looking to voltmeter display connected to the reference load cell (Figure 2, right). The grip force was not controlled and the subjects were asked to maintain a strong grip and full contact between the hand palm and the handle during tests. In order to improve test repeatability each subject is asked to release the handle and relax for $60 \mathrm{~s}$ between each test; this reduces the variability intra-subjects Hewitt, [7]. Even if it is considered not necessary to use a controlled loop feed back system to maintain constant acceleration level on the handle, Hewitt [7], we have used such closed loop control and used a $3 \mathrm{~m} / \mathrm{s}^{2} \mathrm{rms}$ acceleration at the handle for all the tests, so to perform all tests at the same vibration input to the hand. 


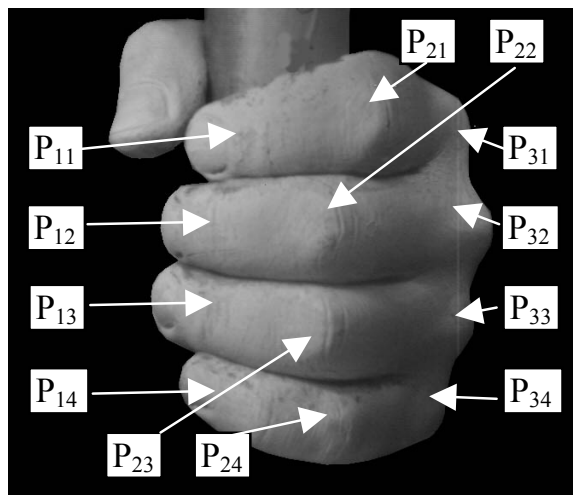

Figure 3: Measurement point $\mathrm{P}_{\mathrm{i}, \mathrm{j}}$ on the hand during grasping.

No temperature and humidity control was performed during the tests. By this procedure, all measurement of the hand vibration will be performed in the direction of the laser beam which is aligned with the shaker vibration axis. Misalignment of accelerometers used to measure transmissibility is a known problem, which can leads up to $20 \%$ of underestimation of the transmissibility with a misalignment of $40^{\circ}$ (Hewitt, [7]).

\section{Results}

The average transmissibility (over 10 measurements) as function of the single frequency excitations, are shown in figure 4 for some of the measured point (all knuckles and distal joints). It is possible to verify how the transmissibility varies as function of the vibration frequency and as function of the measurement point. In our experiment, we measured a maximum average $T R$ of 1.54 (point $\mathrm{P}_{1,3}$, at 90 $\mathrm{Hz}$ ) and a minimum average $T R$ of 0.29 (points $\mathrm{P}_{3,3}$ at $70 \mathrm{~Hz}$ and $\mathrm{P}_{3,4}$ at $90 \mathrm{~Hz}$ ). The average $T R$ over all the measured point is 0.98 and the standard deviation is 0.30 (table 1 and 2).

In figure 5 it is reported the map of average transmissibility (over the 13 subject measured) for the third finger as function of the frequency and of the position along the finger. The higher values of $T R$ are measured on the distal joint at 70 and $90 \mathrm{~Hz}$ (1.14 and 1.54 , respectively); the lower values are at the knuckles at 70 and $90 \mathrm{~Hz}$ (0.29 and 0.32 , respectively).

Figure 6 reports the same analysis of the average transmissibility (over 13 subject) for the fourth finger. Even for this finger the higher values of the $T R$ are in correspondence of the distal joints at 70 and $90 \mathrm{~Hz}(1.41$ and 1.31, respectively); while the lower $T R$ are in correspondence of the knuckle at the frequencies of 70 and $90 \mathrm{~Hz}$ (0.66 and 0.29 , respectively).

The influence of the grip force exerted by the operators during the tests has been investigated asking two subjects to repeat the tests operating both an high grip ( about $90 \%$ of the subject maximum grip) and a low grip (about the $50 \%$ 0f the subject maximum grip). In figure 7 , we report the $T R$ (point $\mathrm{P}_{3,1}$ ) as function 
of the frequency for the 2 subjects and the grip force exerted. It is possible to note how, for the two subjects, the high grip cause an higher $T R$ respect to the low grip for the frequencies 15 and $30 \mathrm{~Hz}$ while there is an inversion (lower TR at high grip) at 70 and $90 \mathrm{~Hz}$.

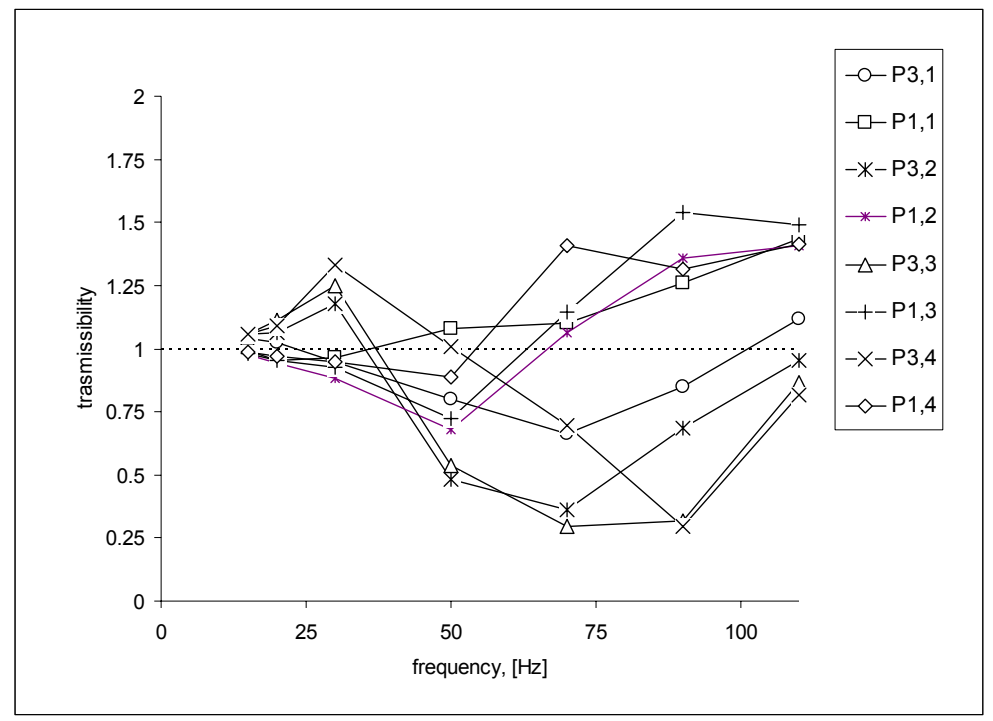

Figure 4: Average transmissibility as function of the excitation frequency for some of the measured points (see figure 3); data from all the 13 subjects.

Table 1: Mean transmissibility values for third and forth finger as function of the frequency.

\begin{tabular}{c|cccccc}
$\begin{array}{c}\text { Finger } \\
\text { position }\end{array}$ & $15 \mathrm{~Hz}$ & $20 \mathrm{~Hz}$ & $30 \mathrm{~Hz}$ & $50 \mathrm{~Hz}$ & $70 \mathrm{~Hz}$ & $90 \mathrm{~Hz}$ \\
\hline $\mathrm{P} 1,3$ & 0.98 & 0.95 & 0.93 & 0.72 & 1.14 & 1.54 \\
$\mathrm{P} 2,3$ & 0.99 & 1.01 & 1.02 & 1.04 & 1.06 & 1.02 \\
P3,3 & 1.06 & 1.11 & 1.25 & 0.54 & 0.29 & 0.32 \\
P1,4 & 0.99 & 0.97 & 0.95 & 0.89 & 1.41 & 1.31 \\
P2,4 & 1.00 & 1.01 & 1.06 & 0.92 & 0.84 & 0.79 \\
P3,4 & 1.06 & 1.09 & 1.33 & 1.01 & 0.66 & 0.29
\end{tabular}

Finally we report in figure 8 the standard deviation of the calculated TR for all the measured point of the 13 subjects as function of the frequency. It appears clear how the dispersion of the data increases for high frequencies. 
Table 2. Standard deviation of the transmissibility values for third and fourth finger as function of the frequency.

\begin{tabular}{c|cccccc}
$\begin{array}{c}\text { Finger } \\
\text { position }\end{array}$ & $15 \mathrm{~Hz}$ & $20 \mathrm{~Hz}$ & $30 \mathrm{~Hz}$ & $50 \mathrm{~Hz}$ & $70 \mathrm{~Hz}$ & $90 \mathrm{~Hz}$ \\
\hline P1,3 & 0.00 & 0.02 & 0.06 & 0.28 & 0.10 & 0.19 \\
P2,3 & 0.01 & 0.01 & 0.00 & 0.29 & 0.14 & 0.19 \\
P3,3 & 0.05 & 0.03 & 0.05 & 0.17 & 0.08 & 0.09 \\
P1,4 & 0.01 & 0.01 & 0.03 & 0.24 & 0.13 & 0.15 \\
P2,4 & 0.02 & 0.01 & 0.02 & 0.39 & 0.18 & 0.09 \\
P3,4 & 0.06 & 0.06 & 0.07 & 0.45 & 0.33 & 0.24
\end{tabular}

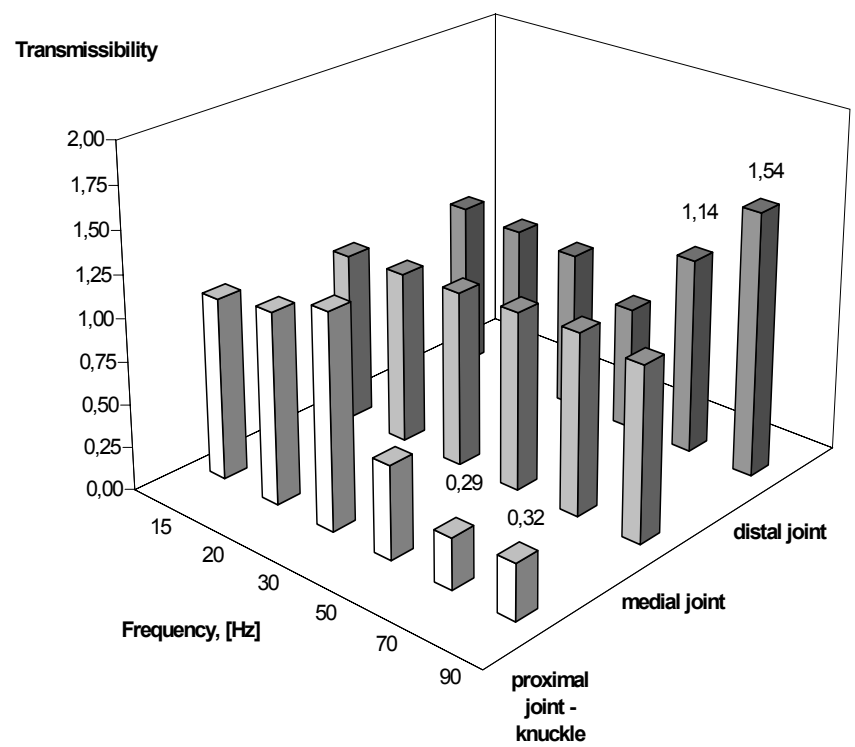

Figure 5: Transmissibility along the third finger as function of the frequency.

\section{Discussion}

The novel method for the measurement of vibrational transmissibility has been proposed, the experimental campaign carried out over 13 subjects has been described and the results are reported.

The method proposed allows to investigate the transmissibility over the fingers, the hand and over other anatomy district (not shown in this paper). Another important feature of the proposed method is the absence of the contact and mass loading effect obtainable by the use of the laser Doppler vibrometer.

The results show a distribution of the measurable acceleration over the fingers joints and the knuckles which demonstrate the difficulty to assign a single 
transmissibility value for one tests if the measurement point is not defined (for example, at $90 \mathrm{~Hz}$ the average TR over the third finger is 0.96 while the max TR is 1.54 on the distal joint).

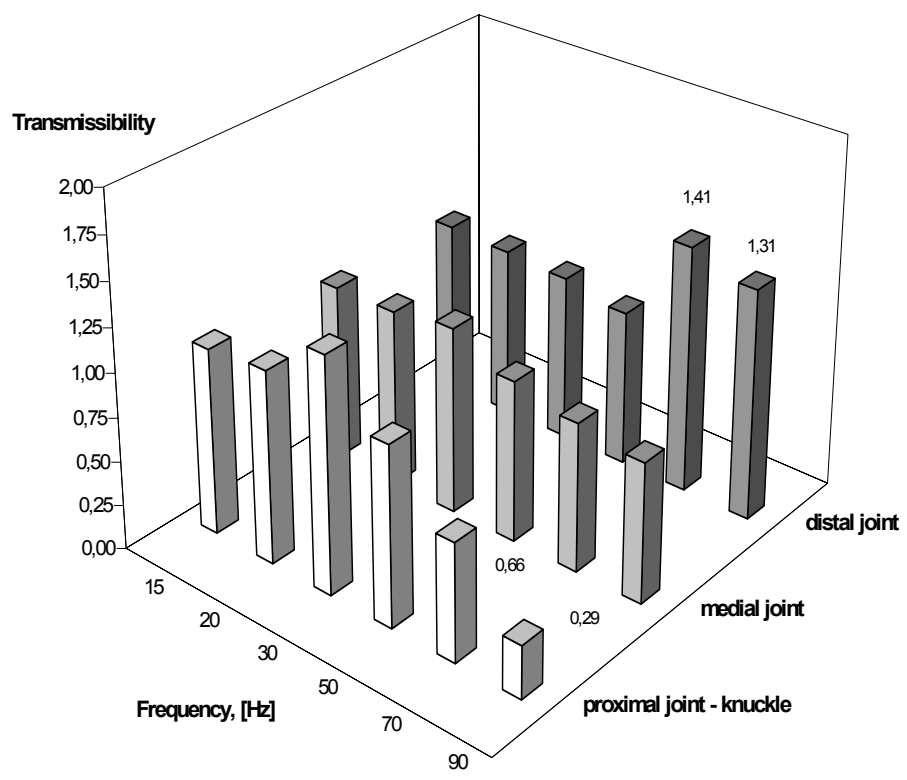

Figure 6: Transmissibility along the fourth finger as function of the frequency.

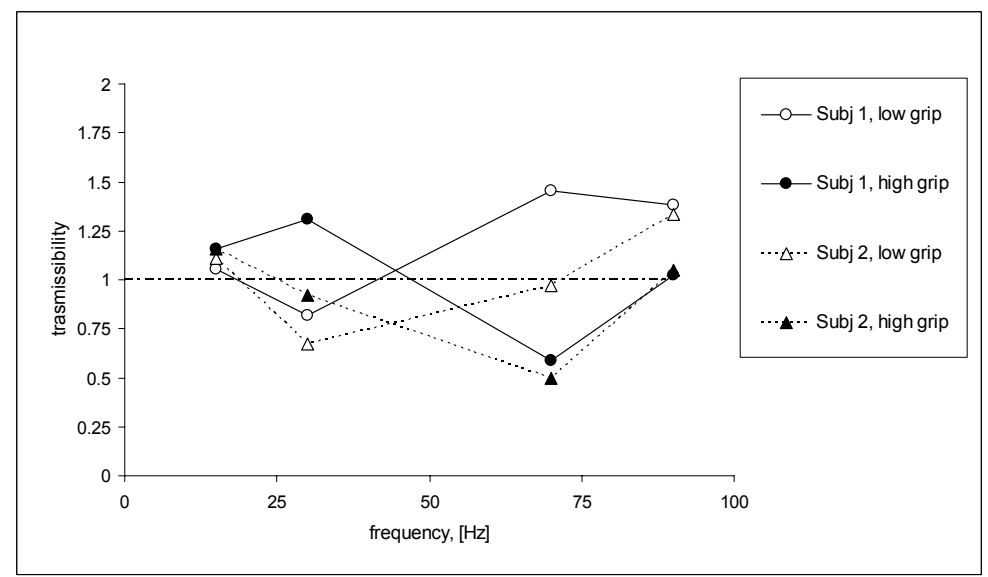

Figure 7: Transmissibility for two subjects as function of the frequency for two level of grip force (low and high). 


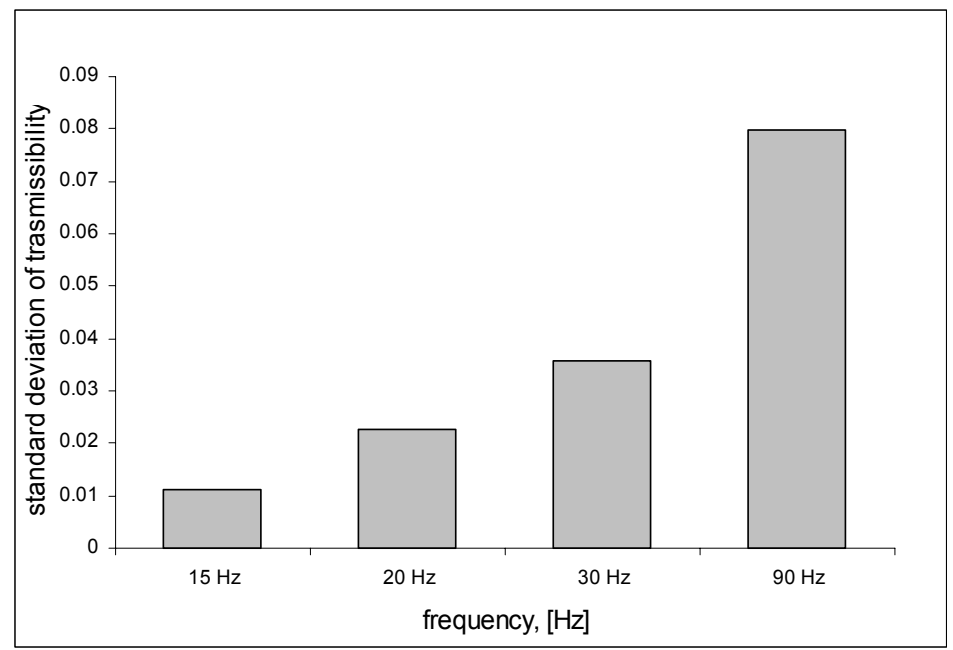

Figure 8: Standard deviation over all transmissibility values as function of the frequency.

The results show a reduction of the transmissibility in correspondence of the knuckles at 70 and $90 \mathrm{~Hz}$ (up to $-71 \%$ ) and an increase of the transmissibility (up to $+54 \%$ ) for the third and fourth distal joints.

As shown by other authors (Hewitt, [7]; Smutz et al. [9]; Dong et al. [11]), there is a clear dependence on frequency of the transmissibility. In particular it has been reported a TR $>1$ for frequencies higher then $50 \mathrm{~Hz}$ for all the distal joints. The third and fourth distal joints showed the highest TR.

$\mathrm{TR}<1$ for frequencies higher then $5 \mathrm{o} \mathrm{Hz}$ have been reported for measurements on all the knuckles with the lowest TR measured on the third and fourth knuckles.

Taking into account the limited number of frequencies tested a resonance on the TR seems to be present at $30 \mathrm{~Hz}$ and a minimum is reported at 70 and $90 \mathrm{~Hz}$ for the knuckles.

Despite the opinion of some other authors (Hewitt, [7]), the effect of the grip force looks to be important (data only from 2 subjects). It is the opinion of the authors, that the influence of such parameter should be considered when transmissibility is measured.

\section{Acknowledgment}

This work as been carried out with the financial support of the Istituto Superiore per la Prevenzione e la Sicurezza del Lavoro - ISPESL (Contract: C17/DIL/02).

\section{References}

[1] M. J. Griffin (1990), Handbook of human vibration. Academic Press, London. 
[2] D. E. Wasserman and J. F. Wasserman, The nuts and bolts of human exposure to vibration, Sound and Vibration, $35^{\text {th }}$ ann issue, Jan 2002.

[3] W. Taylor (1989), The hand-arm syndrome-objective tests. Proc. Inst Phys, 2(9), 425-33.

[4] R. Inaba, S. Mirbod, H. Iwata., Pathophysiology of Vibration-induced White Finger and safety Levels for Hand-Transmitted Vibration, J. Occup Health, 1996, 38, 1-5.

[5] S. Mirbod, R. Inaba and H. Iwata (1997), Operating vibrating tools an prevalence of subjective complaints in vibration syndrome. Cent. Eur. J. Publ. Health, 3, 97-102.

[6] V. Goel and K. Rim (1987), Role of gloves in reducing vibration: An analysis for pneumatic chipping hammer. Am. Ind. Hyg. Assoc. J., 48(1), 9-14.

[7] S. Hewitt (1998), Assessing the performance of anti-vibration gloves - a possible alternative to ISO 10819, 1996. Ann. Occup. Hyg., 42(4), 24552.

[8] T. Shenk and F. Gillmeister, Measurement of hand-arm vibration: errors and uncertainties, Proc. of $9^{\text {th }}$ Int Conf on Hand-Arm Vibration, 5-8 June 2001, Nancy.

[9] W. P. Smutz, R. Dong, B. Han, A. W. Schopper, D. E. Welcome and M. L. Kashon, A Method for Reducing Adaptor Misalignment when Testing Gloves Using ISO 10819, Ann. Occup. Hyg., 46(3), 3009-3015, 2002.

[10] ISO 10819:1996-Mechanical vibration and shock-Hand-arm vibration -Method for the measurement and evaluation of the vibration transmissibility of gloves at the palm of the hand. International Organization for Standardization.

[11] R. G. Dong, T. W. McDowell, D. E. Welcome and W. P. Smutz (2005), Correlations between biodynamic characteristics of human hand-arm system and the isolation effectiveness of anti-vibration gloves. Int. J. of Industrial Ergonomics, 35(3), 205-216. 